\title{
Healing by primary closure versus open healing after surgery for pilonidal sinus: systematic review and meta-analysis
}

\author{
Iain J D McCallum, senior house officer, ${ }^{1}$ Peter M King, consultant surgeon, ${ }^{1}$ Julie Bruce, MRC senior research \\ fellow ${ }^{2}$
}

${ }^{1}$ Department of General Surgery, Aberdeen Royal Infirmary

${ }^{2}$ Department of Public Health, University of Aberdeen

Correspondence to: J Bruce, Department of Public Health, University of Aberdeen, School of

Medicine, Aberdeen AB25 2ZD

j.bruce@abdn.ac.uk

doi:10.1136/bmj.39517.808160.BE

\section{ABSTRACT}

Objective To determine the relative effects of open healing compared with primary closure for pilonidal sinus and optimal closure method (midline $v$ off-midline).

Design Systematic review and meta-analyses of randomised controlled trials.

Data sources Cochrane register of controlled trials, Cochrane Wounds Group specialised trials register, Medline (1950-2007), Embase, and CINAHL bibliographic databases, without language restrictions.

Data extraction Primary outcomes were time (days) to healing, surgical site infection, and recurrence rate. Secondary outcomes were time to return to work, other complications and morbidity, cost, length of hospital stay, and wound healing rate.

Study selection Randomised controlled trials evaluating surgical treatment of pilonidal sinus in patients aged 14 years or more. Data were extracted independently by two reviewers and assessed for quality. Meta-analyses used fixed and random effects models, dichotomous data were reported as relative risks or Peto odds ratios and continuous data are given as mean differences; all with 95\% confidence intervals.

Results 18 trials ( $n=1573$ ) were included. 12 trials compared open healing with primary closure. Time to healing was quicker after primary closure although data were unsuitable for aggregation. Rates of surgical site infection did not differ; recurrence was less likely to occur after open healing (relative risk $0.42,0.26$ to 0.66). 14 patients would require their wound to heal by open healing to prevent one recurrence. Six trials compared surgical closure methods (midline $v$ off-midline). Wounds took longer to heal after midline closure than after offmidline closure (mean difference 5.4 days, $95 \%$ confidence interval 2.3 to 8.5 ), rate of infection was higher (relative risk 4.70,95\% confidence interval 1.93 to 11.45 ), and risk of recurrence higher (Peto odds ratio 4.95, 95\% confidence interval 2.18 to 11.24). Nine patients would need to be treated by an off-midline procedure to prevent one surgical site infection and 11 would need to be treated to prevent one recurrence.

Conclusions Wounds heal more quickly after primary closure than after open healing but at the expense of increased risk of recurrence. Benefits were clearly shown with off-midline closure compared with midline closure. Off-midline closure should become standard management for pilonidal sinus when closure is the desired surgical option.

\section{INTRODUCTION}

Pilonidal sinus is a disease that most commonly arises in the hair follicles of the natal cleft of the sacrococcygeal area. Incidence is reportedly 26 per 100000 population, affecting males twice as often as females ${ }^{1}$ and predominantly young adults of working age.

Pilonidal sinus usually presents as an abscess or a chronically discharging, painful sinus tract. Irrespective of the mode of presentation the painful nature of the condition causes significant morbidity, often with a protracted loss of normal activity. The ideal therapy would be a quick cure that allowed patients to return rapidly to normal activity, with minimal morbidity and a low risk of complications.

The management of chronic pilonidal disease is variable, contentious, and problematic. Principles of treatment require eradication of the sinus tract, complete healing of the overlying skin, and prevention of recurrence.

The surgical wound may be left to heal by open healing (secondary intention). Advocates of this technique state that reduced wound tension facilitates trouble free healing without recurrence if all sinus tracts are fully excised. ${ }^{\mathrm{w} 1}$ Alternatively, the wound may be closed to heal by primary closure (primary intention). Methods can be broadly categorised as midline closure techniques (with the wound lying within the natal cleft) or other techniques (where the wound is placed outwith the midline). Advocates of primary closure perceive benefits of faster tissue healing. Variations in current practice reflect the literature, which describes a wide spectrum in patient outcomes for different open and closed surgical techniques.

We determined the relative effects of open healing compared with primary closure for pilonidal sinus and midline versus off-midline wound closure. This paper 
Potentially relevant trials identified and screened $(n=1367)$

Trials excluded: not randomised, abscess data only, or wrong type of surgery $(n=1322)$

Retrieved for more detailed evaluation $(n=45)$

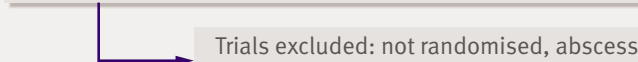

data only, or wrong type of surgery $(n=26)$

Studies included in meta-analysis ( $n=18,+1$ late follow-up)

Fig 1 Flow of studies in systematic review

is based on a Cochrane review published in the Cochrane Library. ${ }^{2}$

\section{METHODS}

We searched the Cochrane Wounds Group specialised register, the Cochrane central register of controlled trials (2006), Medline (1950-2006), Embase (19802006), and CINAHL (1982-2006). A combination of medical subject headings and key words were used: "pilonidal sinus", "fistula", "disease", and "natal cleft". We hand searched relevant conference proceedings and other articles within the Cochrane Wounds Group specialised register. Bibliographies of included studies were reviewed to identify potentially relevant articles. To inquire about unpublished data we contacted authors of included studies.

\section{Inclusion criteria}

Eligible studies were randomised controlled trials comparing two or more surgical techniques for treatment of pilonidal sinus in patients aged 14 years or more. We excluded studies concerning pilonidal abscess, non-randomised trials, paediatric populations, and non-surgical intervention. We made no distinction between presentation of recurrent or de novo disease.

\section{Outcome measures}

Primary outcome measures were time to wound healing, rate of surgical site infection, and recurrence rate. Secondary outcomes were time to return to work, other complications and morbidity, cost, length of hospital stay, and wound healing rate.

\section{Quality assessment and data extraction}

Two reviewers (IJDMcC and JB) independently assessed study quality according to the Cochrane Handbook. ${ }^{3}$ Disagreements were resolved by a third reviewer (PMK). We did not assess blinding after allocation as it was not possible to blind patients, surgeons, or assessors to the intervention. We assessed several criteria: randomisation - whether the sequence was truly random (for example, computer generated, random number tables, coin toss); concealment of allocationwhether a randomisation method described would not allow the investigator or participant to know or influence the intervention group before an eligible participant was entered (allocation concealed); and completeness of follow-up - whether withdrawals were described and reported by treatment group (completeness of follow-up recorded; $>80 \%$ cut-off).

We categorised risk of bias as low (all criteria met), moderate (one or more criteria met), or high (no criteria met).

\section{Statistical analysis}

We defined interventions as closed if primary closure of the skin edges was achieved. If this closure lay in the midline of the natal cleft we allocated the intervention to the midline closure subgroup. When incisions left the closure line lying outside the natal cleft we assigned the intervention to the off-midline closure group. Techniques were deemed to be open if tissue opposition was not achieved; this included techniques such as marsupialisation and open wound healing.

We analysed quantitative data using RevMan. For each outcome we calculated summary estimates of treatment effect (95\% confidence intervals) for each comparison. We calculated mean differences for continuous data, and relative risk or Peto odds ratio with 95\% confidence intervals for dichotomous outcomes. We used the Peto odds ratio when event rates were low. We did not do a sensitivity analysis because of the small numbers of included trials.

We assessed clinical, methodological, and statistical heterogeneity. Statistical heterogeneity was tested using the $\chi^{2}$ test and the $\mathrm{I}^{2}$ statistic. $^{3}$ Fixed effects models were used unless we found significant evidence of statistical heterogeneity or clinical diversity. When diversity or result format precluded meta-analysis, we present the results in a tabular format.

\section{RESULTS}

Of 1367 potentially relevant trials identified and screened, 45 papers were retrieved for full critical appraisal. Eighteen trials were included in the review (fig 1). ${ }^{\text {1-w19 }}$

\section{Study characteristics}

Sample sizes in the trials ranged from 33 to 200 participants (total 1573; table 1). Over $80 \%$ of participants were male. Most studies had been carried out in southern Europe or the Middle East; three trials were from the United Kingdom and the remainder from Norway, Denmark, and Pakistan. The Norwegian group published short term outcomes ${ }^{\mathrm{w} 2}$ and then recurrence data at four years postoperatively. ${ }^{\text {w3 }}$ Data on late recurrence were included once to prevent duplicate data entry.

Only two studies adequately fulfilled all methodological requirements and were considered as having low risk of bias. ${ }^{\text {w14 w17 }}$ Four studies were considered to be at high risk of bias, ${ }^{\mathrm{w} 5 \mathrm{w} 6 \mathrm{w} 12 \mathrm{w} 19}$ and the remaining trials were graded as moderate (table 2).

\section{Characteristics of surgical intervention}

Ten of the 18 studies compared open healing with midline closure techniques, ${ }^{\text {w2-w12 }}$ two compared open 
healing with off-midline closure techniques, ${ }^{\text {wl w13 }}$ and five compared midline closure with off-midline closure techniques. ${ }^{\text {w14-w18 }}$ One study compared off-midline closed techniques (classic rhomboid with asymmetrical rhomboid). ${ }^{\text {w19 }}$ Table 1 gives details of comparisons in each trial.

\section{Time to wound healing}

Time to wound healing (days) was generally defined as full epithelisation over the wound. Ten studies reported this outcome (five reported median data, four reported mean data with standard deviations, and one presented graphical data that could not be interpreted). ${ }^{\text {w10 }}$

\section{Open healing v primary closure}

Owing to inconsistencies in reporting, data on time to wound healing were not pooled, but eligible studies are presented in table 3 . Four trials reported statistically significant quicker (median or mean) time to wound

\section{Table 1 |Characteristics of included studies in systematic review of open healing or primary closure techniques after surgery for pilonidal sinus}

Study Comparison No of participants in group Outcomes

Open healing $v$ midline closure:

\begin{tabular}{|c|c|c|c|c|}
\hline Al-Hassan $1990^{\text {w4 }}$ & Midline closure $v$ open healing & $50 / 50$ & $\begin{array}{l}\text { Time to wound healing, recurrence, length } \\
\text { of stay, return to work, wound healing rate }\end{array}$ & $\begin{array}{l}\text { Mean follow-up of } 33 \text { months for primary } \\
\text { closure and } 25 \text { months for open healing. } \\
>80 \% \text { follow-up }\end{array}$ \\
\hline Füzün $1994^{\text {w5 }}$ & $\begin{array}{l}\text { Midline closure } v \text { Obeid's } \\
\text { technique (open) }\end{array}$ & $55 / 55$ & $\begin{array}{l}\text { Surgical site infection, recurrence, length of } \\
\text { stay, return to work }\end{array}$ & $84 \%$ follow-up over a mean 23 months \\
\hline Gencosmanoglu $2005^{\mathrm{w} 6}$ & $\begin{array}{l}\text { Midline closure } v \text { open healing } \\
\text { (marsupialisation) }\end{array}$ & $69 / 73$ & $\begin{array}{l}\text { Time to wound healing, surgical site } \\
\text { infection, recurrence, other complications, } \\
\text { return to work }\end{array}$ & Complete follow-up at 2 years \\
\hline Hameed $2001^{\mathrm{w} 7}$ & Midline closure $v$ open healing & $23 / 20$ & $\begin{array}{l}\text { Time to wound healing, surgical site } \\
\text { infection, recurrence, cost, other } \\
\text { complications }\end{array}$ & $88 \%$ follow-up at minimum 14 months \\
\hline Khawaja $1992^{\text {w8 }}$ & Midline closure $v$ open healing & $23 / 23$ & $\begin{array}{l}\text { Time to wound healing, recurrence, length } \\
\text { of stay, return to work, other complications }\end{array}$ & $100 \%$ follow-up at 1 year \\
\hline Kronborg $1985^{\text {w9 }}$ & Midline closure $v$ open healing & $66 / 33$ & $\begin{array}{l}\text { Time to wound healing, recurrence, healing } \\
\text { rate }\end{array}$ & $98 \%$ follow-up at 36 months \\
\hline Miocinovic $1999^{\mathrm{w} 11}$ & Midline closure $v$ open healing & $25 / 25$ & Recurrence & $100 \%$ follow-up at 1 year \\
\hline Mohamed $2005^{\mathrm{w} 10}$ & $\begin{array}{l}3 \text { arms: midline closure } v \text { wide } \\
\text { excision and open healing } v \\
\text { limited excision and open } \\
\text { healing }\end{array}$ & $\begin{array}{l}\text { 28/55 (combined open } \\
\text { interventions) }\end{array}$ & $\begin{array}{l}\text { Time to wound healing, length of stay, } \\
\text { recurrence }\end{array}$ & $\begin{array}{l}\text { Two open arms combined for meta-analysis, } \\
\text { follow-up period unclear }\end{array}$ \\
\hline Rao $2001^{\text {w12 }}$ & $\begin{array}{l}\text { Midline closure } v \\
\text { marsupialisation (open) }\end{array}$ & $29 / 30$ & Time to wound healing, pain, healing rate & Follow-up period unclear \\
\hline Sondenaa $1992^{\text {w2 }}$ & Midline closure $v$ open healing & $60 / 60$ & $\begin{array}{l}\text { Time to wound healing, surgical site } \\
\text { infection, return to work, pain, healing rate }\end{array}$ & $\begin{array}{l}\text { Early report of same patient set as Sondenaa } \\
1996^{\text {w3 }}\end{array}$ \\
\hline Sondenaa $1996^{\text {w3 }}$ & Midline closure $v$ open healing & $60 / 60$ & Recurrence & $\begin{array}{l}\text { Final report of Sondenaa } 1992^{\text {w2 }} \text { patient set. } \\
100 \% \text { follow-up at } 3 \text { years }\end{array}$ \\
\hline \multicolumn{5}{|l|}{ Open $v$ closed (off-midline): } \\
\hline Testini $2001^{\text {w1 }}$ & $\begin{array}{l}\text { Karydakis (off-midline } \\
\text { closure) } v \text { open healing }\end{array}$ & $60 / 60$ & $\begin{array}{l}\text { Time to wound healing, recurrence, other } \\
\text { complications, return to work, length of } \\
\text { stay, pain, healing rate }\end{array}$ & $99 \%$ follow-up at minimum 37 months \\
\hline Fazelli $2006^{\text {w13 }}$ & $\begin{array}{l}\text { Z-plasty (off-midline closure) } v \\
\text { open healing }\end{array}$ & $72 / 66$ & $\begin{array}{l}\text { Time to wound healing, recurrence, length } \\
\text { of stay, return to work }\end{array}$ & Mean follow-up 22 months \\
\hline
\end{tabular}

Midline closure $v$ off-midline

closure:

\begin{tabular}{|c|c|c|c|c|}
\hline Abu Galala $1999^{\text {w14 }}$ & $\begin{array}{l}\text { Midline closure } v \text { rhomboid } \\
\text { flap }\end{array}$ & $22 / 24$ & $\begin{array}{l}\text { Surgical site infection, recurrence, length of } \\
\text { stay, return to work, wound healing rate }\end{array}$ & Complete follow-up at 18 months \\
\hline Acka $2005^{\text {w17 }}$ & $\begin{array}{l}\text { Midline closure } v \text { rhomboid } \\
\text { flap }\end{array}$ & $100 / 100$ & $\begin{array}{l}\text { Surgical site infection, recurrence, length of } \\
\text { stay, return to work, other complications, } \\
\text { pain }\end{array}$ & Complete follow-up at 23 months \\
\hline Berkem $2005^{\text {w16 }}$ & $\begin{array}{l}\text { Midline V-Y advancement flap } \\
\text { voff-midline V-Y advancement } \\
\text { flap }\end{array}$ & $16 / 18$ & $\begin{array}{l}\text { Surgical site infection, recurrence, length of } \\
\text { stay }\end{array}$ & Complete follow-up at 30 months \\
\hline Ertan $2005^{\mathrm{w} 15}$ & $\begin{array}{l}\text { Midline closure } v \text { rhomboid } \\
\text { flap }\end{array}$ & $50 / 50$ & $\begin{array}{l}\text { Time to wound healing, surgical site } \\
\text { infection, recurrence, length of stay, return } \\
\text { to work, patient satisfaction, pain }\end{array}$ & $\begin{array}{l}\text { Follow-up } 100 \% \text { at } 3 \text { months but only } 65 \% \text { at } \\
1 \text { year by phone }\end{array}$ \\
\hline Wright $2001^{\mathrm{w} 18}$ & $\begin{array}{l}\text { Bascom (off-midline closure) } v \\
\text { midline closure }\end{array}$ & $16 / 17$ & Recurrence, pain, length of stay & Abstract, follow-up period unclear \\
\hline \multicolumn{5}{|c|}{ Closed (other) $v$ closed (other): } \\
\hline Cihan $2006^{\text {w19 }}$ & $\begin{array}{l}\text { Rhomboid flap } v \text { asymmetrical } \\
\text { rhomboid flap }\end{array}$ & $35 / 33$ & $\begin{array}{l}\text { Surgical site infection, recurrence, length of } \\
\text { stay, return to work, other complications }\end{array}$ & $\begin{array}{l}\text { Follow-up to } 25 \text { months, two unexplained } \\
\text { drop-outs }\end{array}$ \\
\hline
\end{tabular}


Table 2 | Methodological quality assessment of included studies

\begin{tabular}{|c|c|c|c|c|}
\hline Study & Random sequence & Allocation concealed & Follow-up $>80 \%$ & Overall risk of bias \\
\hline Abu Galala $1999^{\text {w14 }}$ & Yes & Yes & Yes & Low \\
\hline Acka $2005^{\mathrm{w} 17}$ & Yes & Yes & Yes & Low \\
\hline Al-Hassan $1990^{\text {w4 }}$ & No & Yes & No & Moderate \\
\hline Berkem $2005^{\mathrm{w} 16}$ & No & Unclear & Yes & Moderate \\
\hline Cihan $2006^{\text {w19 }}$ & No & No & Unclear & High \\
\hline Ertan $2005^{\mathrm{w} 15}$ & Yes & Yes & No & Moderate \\
\hline Fazelli $2006^{\text {w13 }}$ & Yes & No & Yes & Moderate \\
\hline Fuzun $1994^{\text {w5 }}$ & No & No & No & High \\
\hline Gencosmanoglu $2005^{\mathrm{w} 6}$ & No & No & No & High \\
\hline Hameed $2001^{\mathrm{w} 7}$ & Yes & No & Yes & Moderate \\
\hline Khawaja $1992^{\text {w8 }}$ & Yes & Yes & Unclear & Moderate \\
\hline Kronborg $1985^{\text {w9 }}$ & Yes & Yes & No & Moderate \\
\hline Miocinovic $1999^{\text {w11 }}$ & Unclear & No & Yes & Moderate \\
\hline Mohammed $2005^{\text {w10 }}$ & Yes & Yes & Unclear & Moderate \\
\hline Rao $2001^{\text {w12 }}$ & Unclear & Unclear & Unclear & High \\
\hline Sondenaa $1992^{\mathrm{w} 2}$ & Yes & No & Yes & Moderate \\
\hline Sondenna $1996^{\mathrm{w} 3}$ & Yes & No & Yes & Moderate \\
\hline Testini $2001^{\mathrm{w} 1}$ & Yes & Yes & Unclear & Moderate \\
\hline Wright $2001^{\text {w18 }}$ & Yes & Yes & Unclear & Moderate \\
\hline
\end{tabular}

healing after primary closure. ${ }^{\mathrm{w} 2 \mathrm{w} 6 \mathrm{w} 7 \mathrm{w} 12}$ Three studies also showed quicker healing in the primary closure group although no formal statistical tests were reported (table 3). ${ }^{\text {w w8 w9 }}$ For the one study using off-midline closure (Z-plasty), a significantly shorter time to healing in the Z-plasty group was found (41 days, 95\% confidence interval 20 to 160 v 15.4 days, 10 to 34 ; $\mathrm{P}<0.001)$. ${ }^{\mathrm{w} 13}$

Midline closure $v$ off-midline closure

One trial (100 participants) reported that midline wounds took significantly longer to heal than offmidline rhomboid flaps (mean difference 5.4 days, 95\% confidence interval 2.3 to 8.5 days). ${ }^{\text {w15 }}$

Rate of surgical site infection

Open healing v primary closure

Five trials (559 participants) assessed the rate of surgical site infection after open healing compared with primary closure (all techniques). ${ }^{\text {2 w5-w7 w13 }}$ Infection rates were marginally higher after open healing; however, this was not statistically significant $(1.20$,
Study or subcategory

Open healing $\mathbf{v}$ midline closure

Sondenaa $1992^{\text {w2 }}$

Fuzun $1994^{\text {w5 }}$

Hameed 2001 w7

Gencosmanoglu $2005^{\text {w6 }}$

Subtotal $(95 \% \mathrm{Cl})$

Test for heterogeneity: $\chi^{2}=6.15, \mathrm{df}=3, \mathrm{P}=0.10, \mathrm{I}^{2}=51.2 \%$

Test for overall effect: $\mathrm{z}=0.36, \mathrm{P}=0.72$

Open healing $\boldsymbol{v}$ closed (other)

Fazelli $2006^{\text {w13 }}$

Subtotal $(95 \% \mathrm{Cl})$

Test for heterogeneity: not applicable

Test for overall effect: $z=0.77, P=0.44$

Total $(95 \% \mathrm{Cl})$

Test for heterogeneity: $\chi^{2}=6.06, \mathrm{df}=4, \mathrm{P}=0.19, \mathrm{I}^{2}=34.0 \%$

Test for overall effect: $\mathrm{z}=0.45, \mathrm{P}=0.65$

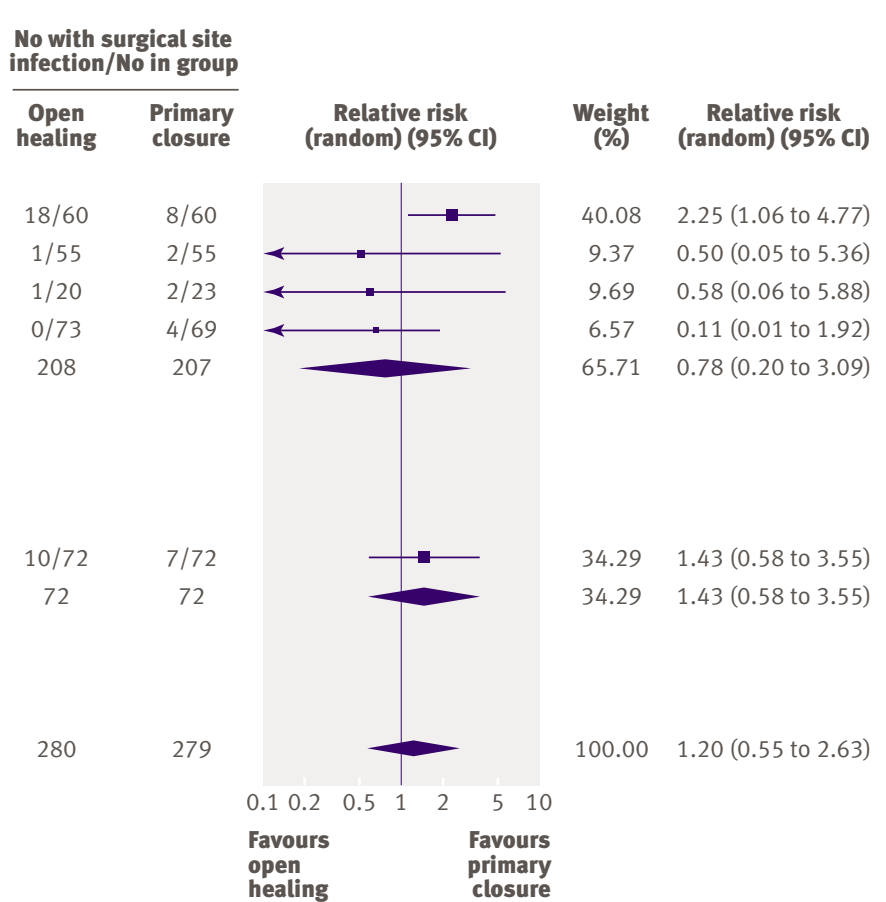


Table 3 | Time to wound healing using open healing compared with midline closure techniques

\begin{tabular}{|c|c|c|c|c|c|c|}
\hline \multirow[b]{2}{*}{ Study } & \multicolumn{2}{|c|}{ Sample size } & \multicolumn{2}{|c|}{$\begin{array}{l}\text { Time (days) to wound healing } \\
\text { (range) }\end{array}$} & \multirow[b]{2}{*}{$P$ value } & \multirow[b]{2}{*}{ Result format } \\
\hline & Open healing & Midline closure & Open healing & Midline closure & & \\
\hline Gencosmanogluw6 & 73 & 69 & $79(21-112)$ & $14(14-63)$ & $<0.001$ & Median \\
\hline Kronborg $^{\text {w9 }}$ & 33 & 32 & $64(17-157)$ & $13(7-203)$ & NR & Median \\
\hline $\mathrm{Rao}^{\mathrm{w} 12}$ & 30 & 29 & $61(34-132)$ & $27(24-68)$ & $<0.001$ & Median \\
\hline Khawaja $^{\text {w8 }}$ & 23 & 23 & 41 (NR) & 14 (NR) & NR & Median \\
\hline Al-Hassan w4 & 40 & 42 & $91(28-546)$ & $10(10-15)$ & NR & Mean \\
\hline Sondenaa $^{\text {w2 }}$ & 59 & 60 & $70(28-266)$ & $14(14-112)$ & $<0.001$ & Median \\
\hline Hameed $^{\mathrm{w7}}$ & 20 & 23 & 70 (59-91) & $15(12-21)$ & $<0.05$ & Mean \\
\hline
\end{tabular}

$\mathrm{NR}=$ not reported

0.55 to 2.63 ; fig 2). A single study comparing open healing with Z-plasty reported a non-significant increase in infection after open healing $(1.43,0.58$ to 3.55). ${ }^{\text {w13 }}$

Overall, the rate of surgical site infection after pilonidal surgery was low, except for two studies where rates with open healing were $14 \%$ and $22 \%$. ${ }^{\text {w2 w13 }}$

\section{Midline closure $v$ off-midline closure}

Four trials (380 participants) assessed surgical site infection $^{\text {w14-w17}}$; infection rates varied from $0 \%{ }^{\text {w16 }}$ to $13 \%{ }^{\text {w15 }}$ Overall, rates of infection were significantly higher after midline closure $(4.70,1.93$ to 11.45 ; fig 3$)$. Nine patients would need to be treated by an offmidline closure procedure to prevent one surgical site infection.

The trial comparing two off-midline procedures $(68$ participants) reported significantly fewer infections after modified asymmetrical flap (3\%) than after classic rhomboid flap $(23 \% ; \mathrm{P}=0.03) .{ }^{\text {w19 }}$

\section{Recurrence rate}

Recurrence rate was the most commonly recorded outcome (18 trials). As timing of follow-up of recurrence varied widely it was categorised as less than or greater than one year and grouped by proportion followed up (greater than or less than $80 \%$ ). A Peto odds ratio was used for the comparison of midline closure versus off-midline closure owing to the low event rate.

\section{Open healing v primary closure}

Data on recurrence were reported in 11 trials (994 participants), 10 of which had high rates of follow-up $(>80 \%) .{ }^{\text {w1 w2 w4-w11 w13 }}$ Recurrence of sinus was a rare outcome (affecting 81 of 994 participants, 8\%) and several trials failed to detect any events. Meta-analysis indicated that open healing of pilonidal sinus is associated with a $58 \%$ lower risk of recurrence than primary closure $(0.42,0.26$ to 0.66 ; fig 4$)$. Fourteen patients would require their wound to heal by open healing to prevent one recurrence.

Pooling of the eight trials that compared open healing with midline closure showed a statistically significant lower recurrence rate with open healing (relative risk $0.39,0.23$ to 0.66 ; fig 4 ).

Two trials (238 participants) compared open healing with off-midline closure; off-midline closure was by Karydakis flap in one ${ }^{\mathrm{w} 1}$ and by Z-plasty in the other. ${ }^{\text {w13 }}$ A random effects model was used for analysis owing to differing surgical technique; no significant difference was shown $(0.70,0.20$ to 2.42 ; fig 4$)$.

\section{Midline closure v off-midline closure}

Five trials that assessed rate of recurrence (413 participants) provided data on 24 events (5.8\%). ${ }^{\text {w14-w18 }}$ Overall, the recurrence rate was significantly higher after midline closure (Peto odds ratio 4.95, 2.18 to 11.24; fig 5). This equates to 11 patients requiring offmidline closure to prevent one recurrence.

One study reported two recurrences after classic

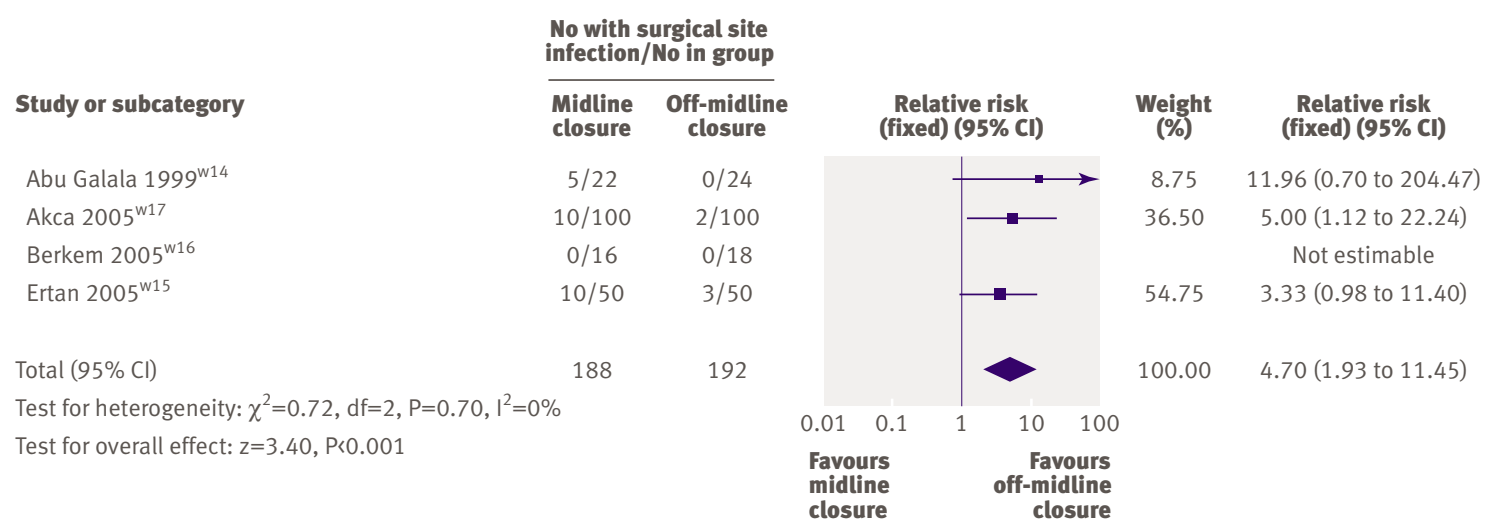


rhomboid flap and none after modified rhomboid flap in a group of 68 participants (odds ratio 5.00, 0.23 to 108.13). ${ }^{\text {19 }}$ Such large confidence intervals prevented meaningful interpretation of these data.

\section{Time to return to work}

Eleven trials reported time to return to work as an outcome, ${ }^{\mathrm{w} 1 \mathrm{w} 2 \mathrm{w} 4 \mathrm{w} 5 \mathrm{w} 13-\mathrm{w} 15 \mathrm{w} 19}$ two of these did not report variance values and could not be included in the metaanalysis. $^{\mathrm{w} 4 \mathrm{w} 13}$ Three trials reported medians (interquartile ranges), hence their data could not be pooled. ${ }^{\mathrm{w} 6 \mathrm{w} 8 \mathrm{w} 17}$

\section{Open healing v primary closure}

Of five trials reporting time to return to work (563 participants); three used midline closure ${ }^{\mathrm{w} 2 \mathrm{w} 4 \mathrm{w} 5}$ and two off-midline closure. ${ }^{\mathrm{w} 1 \mathrm{w} 13}$ Patients undergoing excision with open healing took longer to return to work than those having closed operations regardless of closure method (open $v$ closed (all) mean difference 10.48 days, 5.75 to 15.21 ; fig 6 ), midline closure (8.56 days, 2.97 to
14.15; fig 6$)$, or off-midline closure (15.30 days, 6.44 to 24.16; fig 6).

\section{Midline closure v off-midline closure}

In two studies reporting mean time to return to work no overall effect was shown (1.68 days, -19.59 to 22.94). ${ }^{\mathrm{w} 14 \mathrm{w} 15}$ The studies had opposing findings: the trial $^{\text {w14 }}$ suggesting longer time after off-midline closure was of higher quality.

One study comparing modified asymmetrical rhomboid flap with classic rhomboid flap found a difference of only two days in return to work, favouring the asymmetrical modified Limberg flap (9.3 days (SD 0.34) v 11.7 (SD 0.45); $\mathrm{P}<0.001) .{ }^{\mathrm{w} 19}$

\section{Other complications and morbidity}

Numerous different complications were reported by the trials, including maceration, primary failure, wound dehiscence, haematoma, early suture removal, and flap oedema. ${ }^{\text {w1 }}$ w2w4 w6-w8 w13 w15w17w19 Owing to heterogeneity of outcomes and reporting of outcomes (for example, number of events and number of patients with events), a random effects model was selected.

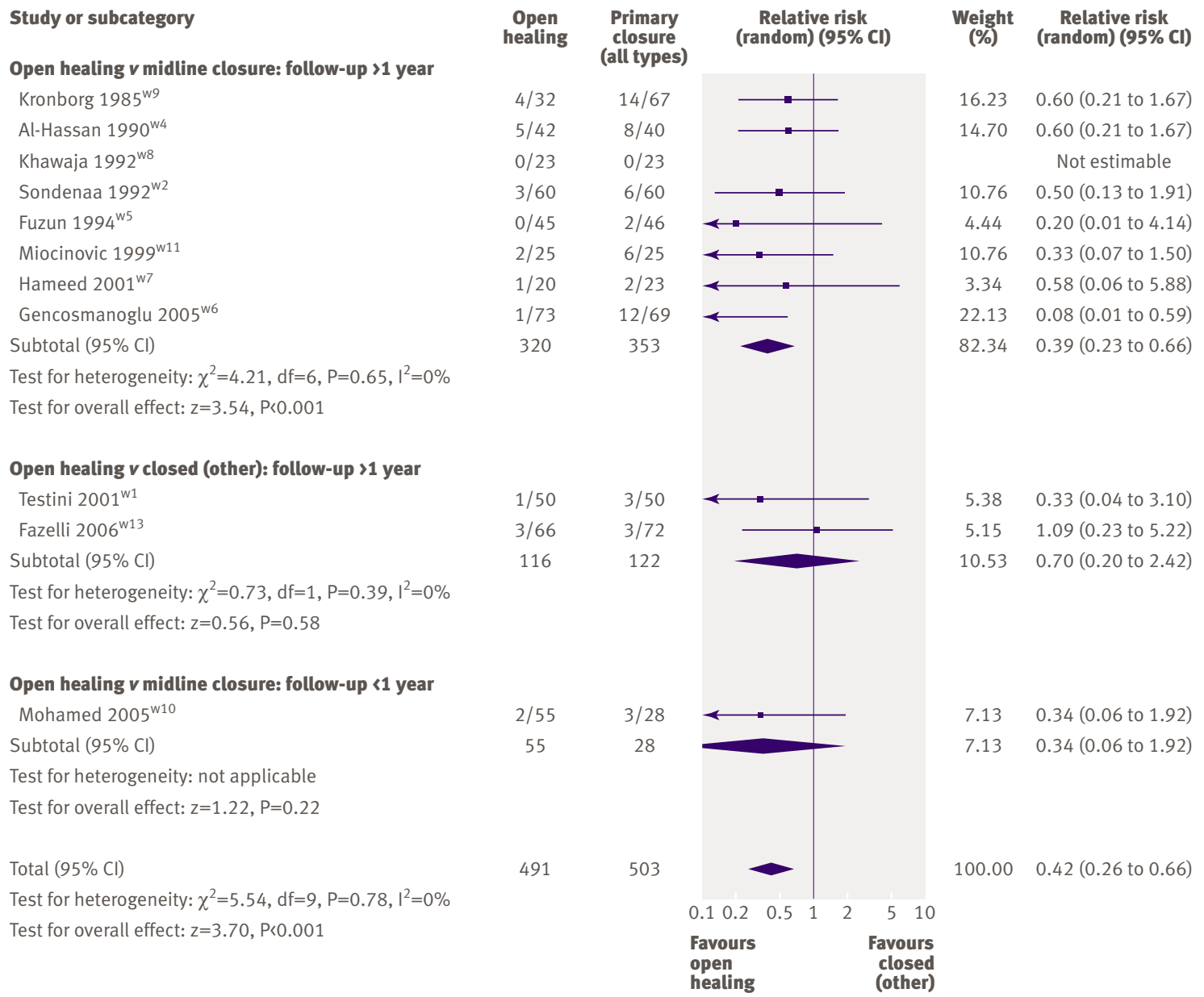


Study or subcategory

Follow-up >1 year ( $\mathbf{8 0 \% )}$

Abu Galala $1999^{\text {w14 }}$

Akca $2005^{\text {w17 }}$

Subtotal $(95 \% \mathrm{Cl})$

Test for heterogeneity: $\chi^{2}=0.00, \mathrm{df}=1, \mathrm{P}=0.98, \mathrm{I}^{2}=0 \%$

Test for overall effect: $z=3.72, P<0.001$

Follow-up $<1$ year ( $\$ 80 \%)$

Wright $2001^{\text {w18 }}$

Subtotal $(95 \% \mathrm{Cl})$

Test for heterogeneity: not applicable

Test for overall effect: $z=1.39, P=0.16$

Follow-up $>1$ year ( $(80 \%)$

Berkem 2005 ${ }^{\text {w16 }}$

Ertan $2005^{\text {w15 }}$

Subtotal $(95 \% \mathrm{Cl})$

Test for heterogeneity: $\chi^{2}=0.17, \mathrm{df}=1, \mathrm{P}=0.68, \mathrm{I}^{2}=0 \%$

Test for overall effect: $z=2.44, P=0.01$

Total $(95 \% \mathrm{Cl})$

Test for heterogeneity: $\chi^{2}=7.27, \mathrm{df}=4, \mathrm{P}=0.12, \mathrm{I}^{2}=45.0 \%$

Test for overall effect: $z=3.82, P<0.001$

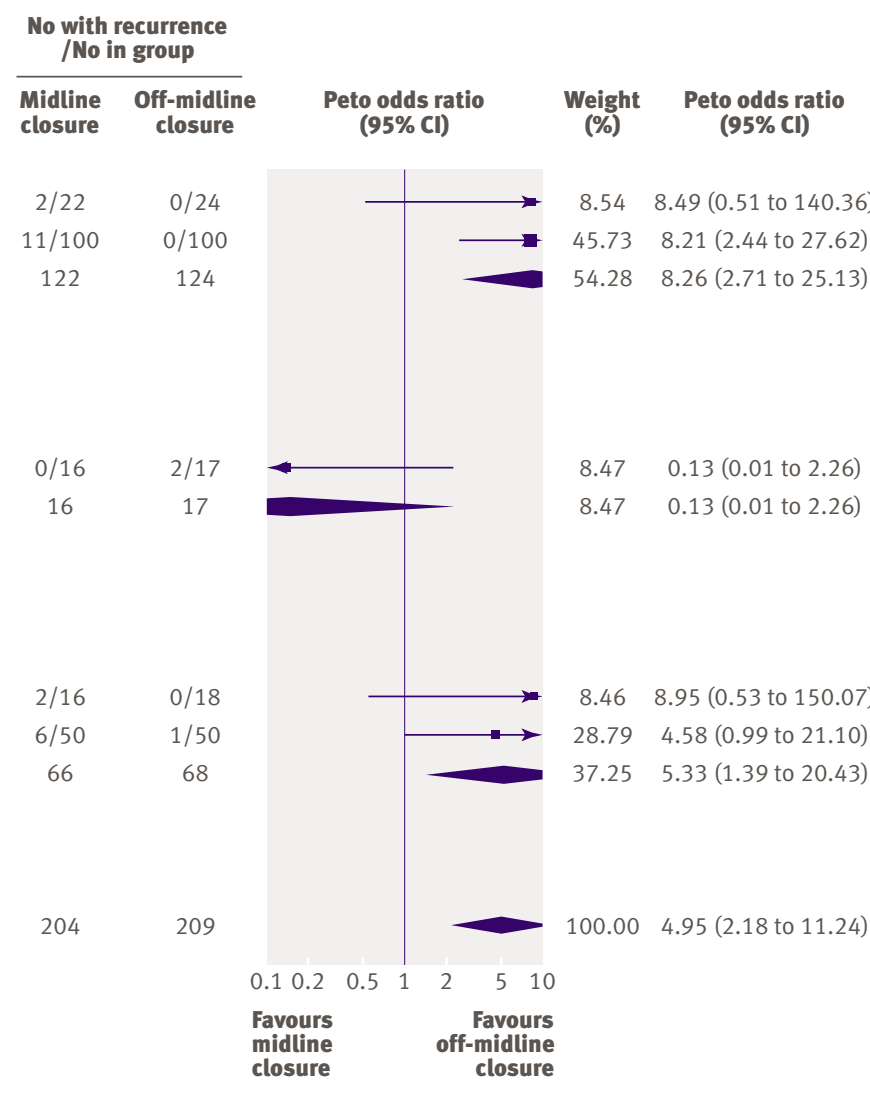

Fig 5 Recurrence rate of pilonidal sinus after surgery using midline closure or closed (other) procedures

Open healing v primary closure

Of seven trials reporting data on 688 patients, ${ }^{\text {w1 }}$ w2 w4 w6w8 ${ }^{13}$ the rate of complications did not differ $(0.67,0.27$ to 1.70 ; fig 7 ).

Five of these trials (433 participants) compared open healing with midline closure; no significant difference was found in rate of postoperative complications $(0.56$, 0.12 to 2.51 ; fig 7 ). Two trials (244 participants) compared open healing with off-midline closure (Zplasty and Karydakis flap); no significant difference was shown $(0.87,0.36$ to 2.10$)$. $^{\text {w1 w13 }}$

\section{Midline closure v off-midline closure}

Two trials reported complications with off-midline rhomboid flaps compared with midline closure. ${ }^{\text {w14 w15 }}$ A clear benefit was shown with off-midline closure $(8.94,2.10$ to 38.02$)$. One study reported a higher complication rate (dehiscence) after classic rhomboid flap (8/35 patients, $23 \%)$ than after modified asymmetrical flap $(1 / 33,3 \% ; \mathrm{P}=0.03) .{ }^{\text {w19 }}$

\section{Patient satisfaction}

A small but statistically significant preference was shown for off-midline closure than for midline closure, where satisfaction was measured using a 0-10 visual analogue scale in the single study that measured this outcome. ${ }^{\text {w15 }}$ A mean difference of one point on a visual analogue scale is, however, unlikely to be translated into a meaningful clinical difference (7.4 (SD 1.4) v 6.5 (SD 1.7) visual analogue scale; $\mathrm{P}<0.001)$.
Cost

Only one trial, from Pakistan, reported data on cost and found that midline closure was cheaper than open healing. ${ }^{\text {w7 }}$ This finding may not be generalisable to most other healthcare systems.

\section{Length of hospital stay}

Open healing v primary closure

Reporting on length of hospital stay varied across studies owing to differing criteria for discharge. Reporting was unclear ${ }^{\text {w18 }}$ or could not be included in a meta-analysis. ${ }^{\text {w4 } 5}$ Five trials (533 participants) assessed length of hospital stay, ${ }^{\mathrm{w} 1 \mathrm{w} 4 \mathrm{w} 5 \mathrm{w} 10 \mathrm{w} 13}$ three of which used a closed midline procedure. ${ }^{\mathrm{w} 4 \mathrm{w} 5 \mathrm{w} 10}$ Overall, a trend towards a shorter stay was shown in those having open procedures compared with closed procedures; however, this was not significant (mean difference -1.26 days, $95 \%$ confidence interval -2.77 to 0.24 ; fig 8). A random effects model was used, as significant statistical heterogeneity was shown within the results $\left(\mathrm{I}^{2}=98.2 \%\right)$. Data were pooled as the high statistical heterogeneity was caused by the result of one study, ${ }^{\text {w1 }}$ where both operations were carried out on a day case basis compared with other studies where samples were inpatients.

\section{Midline closure $v$ off-midline closure}

Two trials found that length of stay was significantly shorter after off-midline closure than midline closure (mean difference 1.95 days, 95\% confidence interval 
0.21 to 3.69; fig 9). A random effects model was used as there was evidence of statistical heterogeneity $\left(\mathrm{I}^{2}=72.3 \%\right)$.

\section{Pain}

Postoperative pain was reported in binary and continuous formats: as proportion or rate of patients experiencing pain and mean or median pain scores per group.

\section{Open healing v primary closure}

Three studies reported pain. ${ }^{\text {w1 w2 w12 }}$ Median (range 0100) pain scores on postoperative day 4 were significantly lower for patients having closed procedures $(10(0-73) v 35(0-63) ; \mathrm{P}<0.05) .{ }^{\text {w12 }}$ For two studies (220 participants) rate of pain did not differ between open and closed methods (1.13, 0.43 to 2.80$).{ }^{\text {w1 w2 }}$ One of the studies compared midline closure with open healing and showed identical rates of pain $(10 \%)$ in both groups in the early postoperative period. ${ }^{\mathrm{w} 2}$ The other study compared open healing with Karydakis flap and showed similar rates of acute pain in both groups (closed $4 \%$ v open $6 \%$ ). ${ }^{\mathrm{w} 1}$

\section{Midline closure $v$ off-midline closure}

Two studies measured postoperative pain. ${ }^{\mathrm{w} 15 \mathrm{w} 18}$ Mean pain scores were only estimable for one of the trials ${ }^{\mathrm{w} 15}$ as no variance data were obtained for the trial that assessed the Bascom flap method. ${ }^{\mathrm{w} 18}$ Improved pain scores, using the short form-36 pain domain (0-100), were reported after off-midline closure although this was not significant (mean difference $-13.00,-19.41$ to $-6.59) .{ }^{\text {w15 }}$

\section{Wound healing rate}

Open healing v primary closure

Five trials (474 participants) reported rates for wound healing after open healing and primary closure (all techniques); overall no difference was found in wound healing rate by type of closure $(0.94,0.84$ to 1.05$) .{ }^{\text {w1 w2 }}$ w4 w9 w12

No significant difference was observed in the four studies comparing open healing with midline closure ${ }^{\mathrm{wl}}$ w2 w4 w9 (fig 10) or in the single study (100 participants) examining open healing with off-midline closure $(1.02$ 0.98 to 1.06$).^{\mathrm{w} 1}$

\section{Midline closure $v$ off-midline closure}

One study compared healing rates after midline closure with those after off-midline closure (rhomboid flap). ${ }^{\text {w14 }}$ A statistically significant improvement was shown in healing rates after off-midline closure $(0.77$, 0.62 to 0.97$)$.

\section{DISCUSSION}

After surgery for pilonidal sinus wounds healed more quickly when primary closure was used but the risk of sinus recurrence was higher than with open healing. No significant difference was found in rate of surgical site infection between the two approaches. A careful assessment of the harms and benefits of each should be made when considering surgical treatment. A clear benefit was, however, found with off-midline closure compared with midline closure.

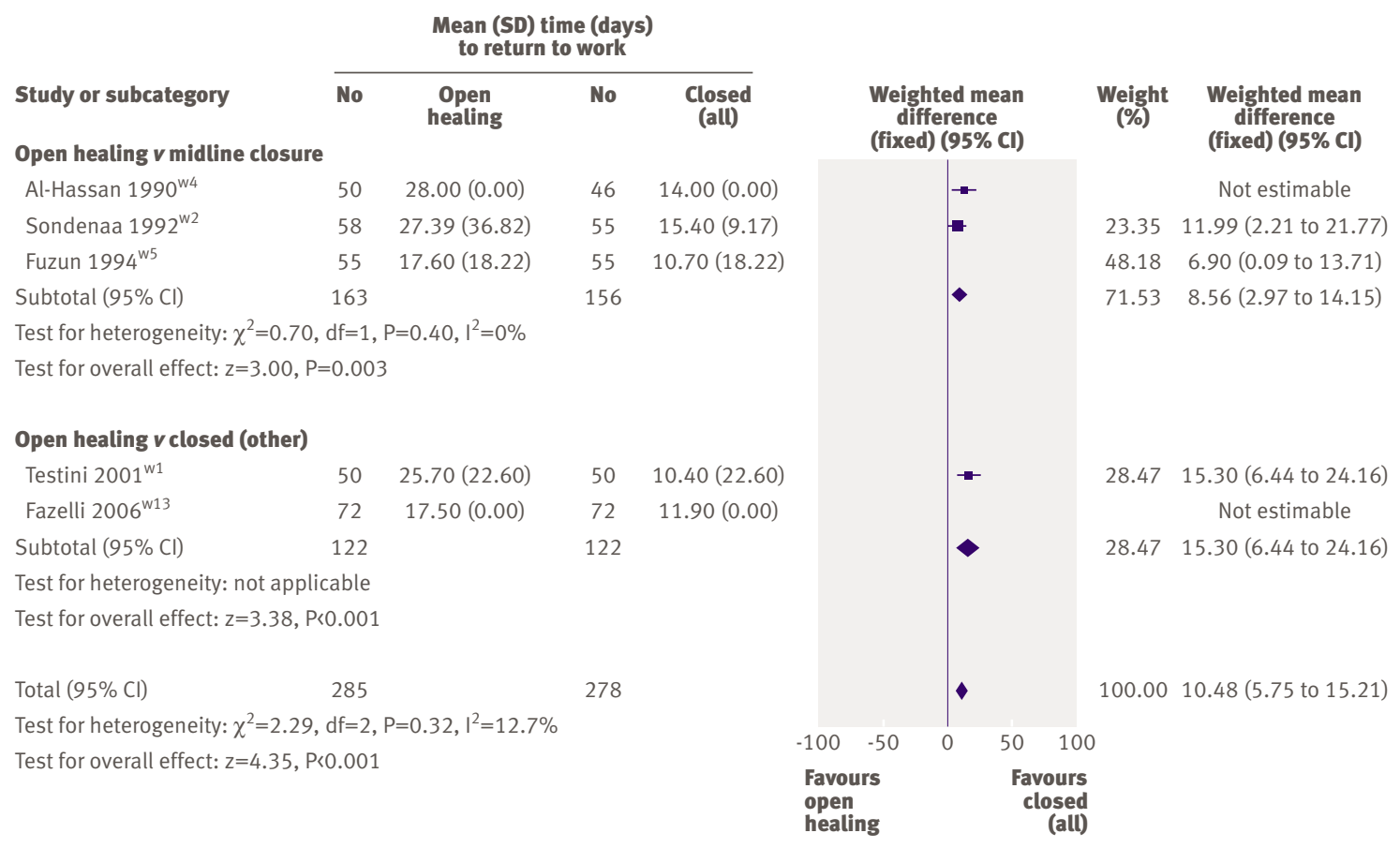




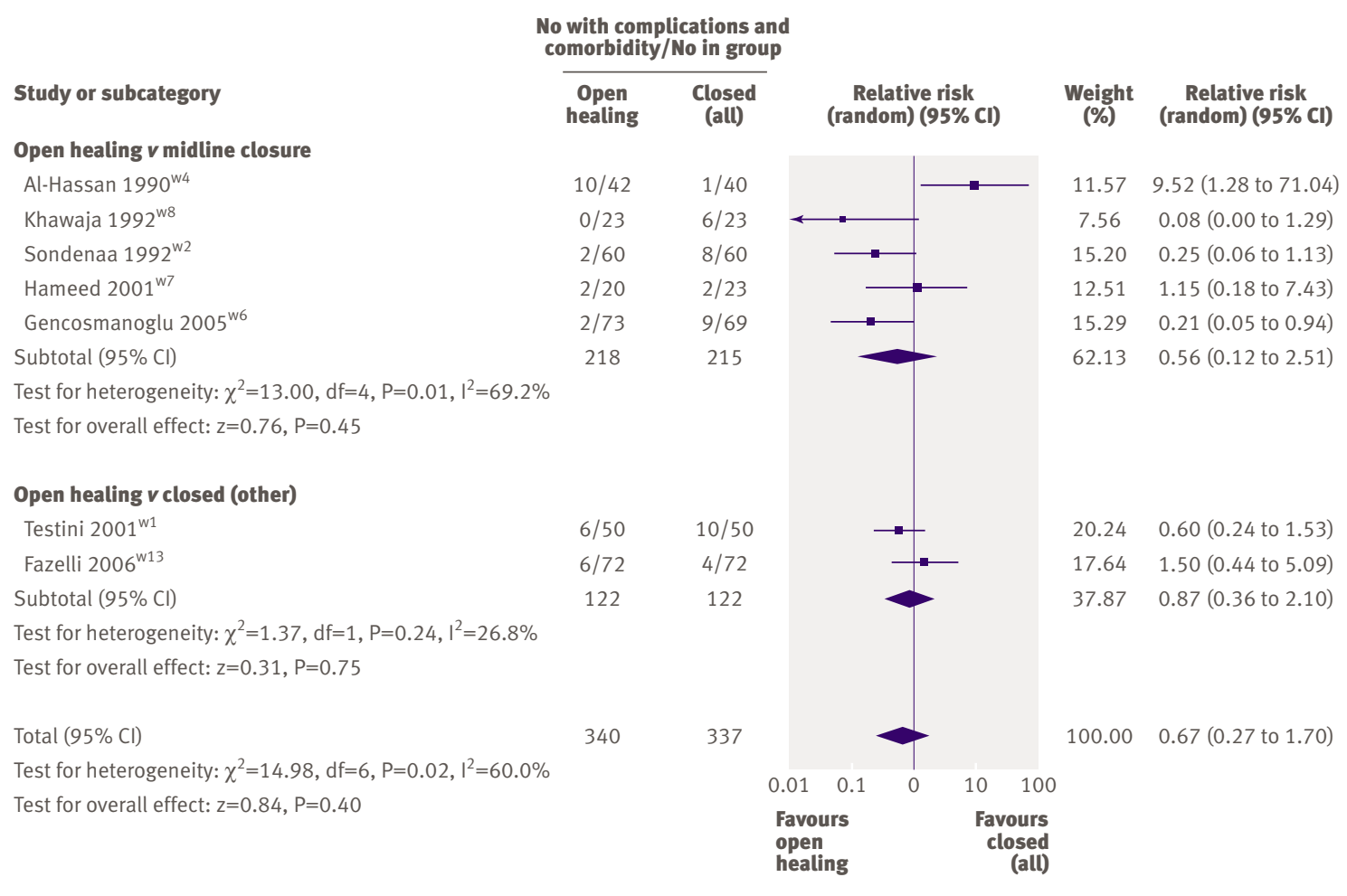

Fig 7 Complications and morbidity after pilonidal surgery using open healing or primary closure
Overall, recurrence of pilonidal sinus was more common with midline closure $(59 / 503,11.7 \%)$ than with open healing $(22 / 491,4.5 \%)$ which showed a $58 \%$ lower risk of recurrence at one year postoperatively. This equates to 14 patients experiencing recurrence per 100 undergoing primary closure (5 per 100 undergoing open healing). It is unsurprising that open wounds take longer than closed wounds to heal, and this reflects on other outcomes such as length of hospital stay and time taken to return to work. Early return to normal activity is an important outcome, and a clear benefit was found in patients undergoing primary closure. The general consensus in the surgical literature is that open healing results in a lower rate of infection at surgical sites; evidence from our systematic review is inconclusive to support this assumption. Postoperative infection was not recorded by all trials and the small number of events prevented us from accurately estimating an effect size. Despite differences in wound healing time, rate of infection and other postoperative complications did not differ between open healing and primary closure.

When the choice of treatment for pilonidal sinus was excision and primary closure our review found significant benefit after off-midline closure. Fewer infections, recurrences, and other complications occurred and wound healing was quicker after offmidline closure than after midline closure. Recurrence rates were significantly lower $(1.4 \%)$ than with midline closure (10.3\%); this finding was previously reported in a non-systematic review, where fewer recurrences occurred after off-midline procedures $(1.6 \% v 9.4 \%){ }^{4}$
Our systematic review identified an infection rate of $10.4 \%$ after midline closure and $6.3 \%$ after off-midline closure, which are similar to the aggregated rates $(12.4 \%$ v $7.6 \%)$ reported by one study. ${ }^{4}$ Although that review aggregated rates for infection, early failure, and recurrence by different closure methods (midline, asymmetrical or oblique; rhomboid; VY-plasty and Z-plasty), this study included results from case series and retrospective surgical audits and failed to assess the methodological quality of individual studies.

Pilonidal sinus predominantly affects younger populations and therefore this disease has an economic impact. To date this has not been formally evaluated. Time to return to work is a function of several other variables, including time to wound healing, pain, wound complications, wound breakdown, and other management factors. No benefit was specifically shown for time to return to work after off-midline closure, although significant heterogeneity existed between the included studies. This is an area worthy of further evaluation.

\section{Limitations}

Owing to the nature of the surgical treatment for pilonidal sinus, blinding of surgeons, patients, and assessors is not possible and some risk of bias exists; therefore results must be interpreted with caution. Many small variations in surgical technique occur, including depth and extent of dissection, undermining of tissues, and type of suture materials used. We have attempted to group interventions to maintain clinical relevance whenever possible. This, as in all meta- 


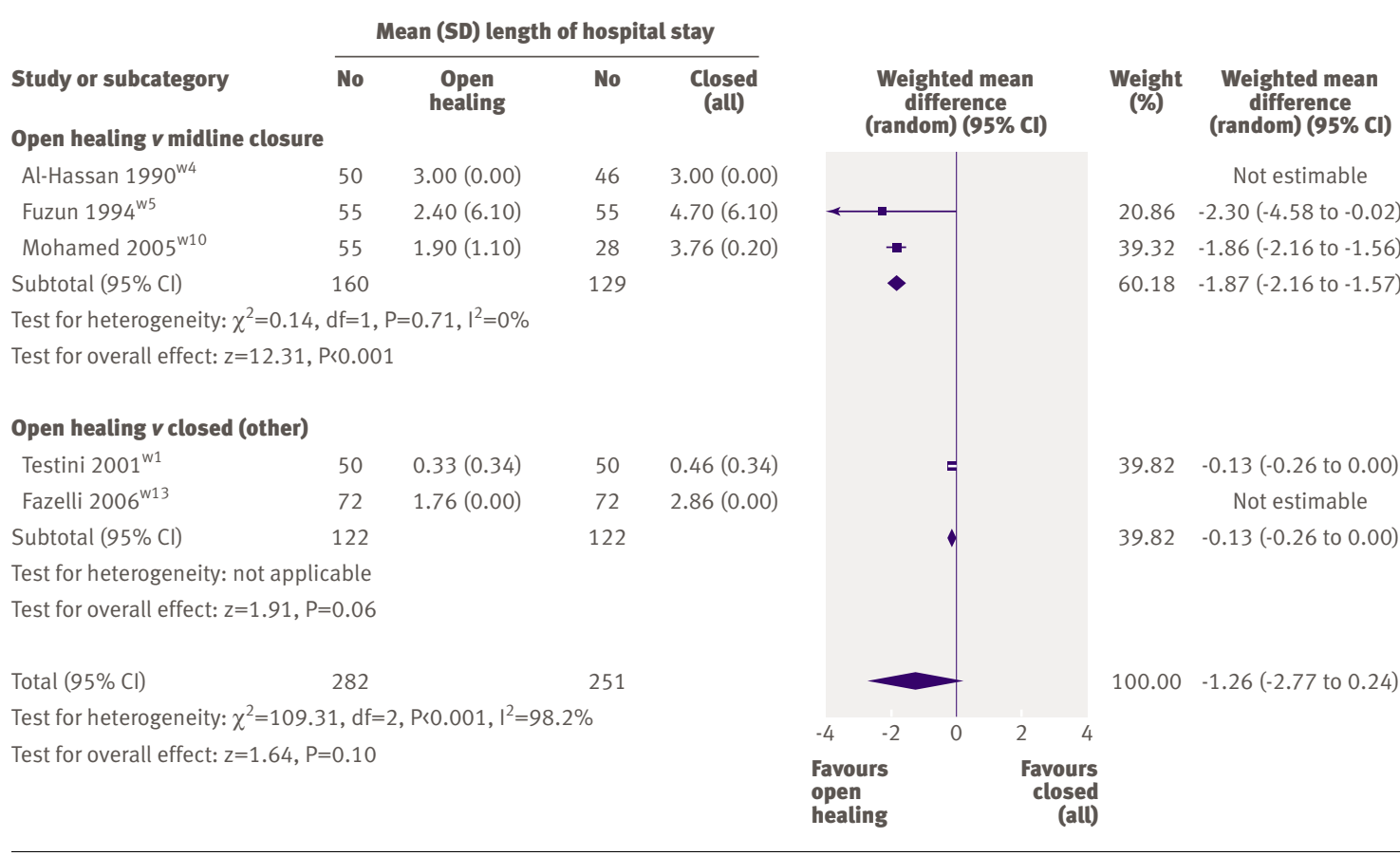

Fig 8 Length of hospital stay after pilonidal surgery using open healing or primary closure analyses, represents a compromise, which has to be reached to provide meaningful comparison. Study groups contain variable interventions, however, and the optimal specific technique for differing severities of disease cannot be inferred from the data presented here. Although cointerventions were reported in some trials (for example, antibiotic prophylactic regimens, antibiotic wound impregnation, wound dressings), it was not possible to incorporate these factors into subgroup comparisons.

\section{Methodological quality}

Seventeen studies were original reports of primary data, one study was published as a conference abstract, and one group reported outcome data for a cohort recruited four years earlier. We did not undertake formal assessment for publication bias. The methodological quality of the studies was disappointing; most trials were small and at risk of failing to detect clinically relevant differences as statistically significant. Certain important postoperative outcomes, such as recurrence and infection, are rare outcomes and this may lead to imprecise estimation in studies with small sample sizes. Other methodological flaws such as poor randomisation techniques and inadequate follow-up further limit the interpretation of findings. Few studies provided definitions of postoperative events, therefore trials should include and report standard measures to allow objective and comparable assessment of outcomes.

Standardisation was lacking in reporting of outcomes, length of trial, and proportion of recruited sample followed up; many trials failed to accurately record or present this information. None of the trials reported follow-up beyond four years, and late recurrence is not represented here. Recurrence may also be underestimated slightly as some studies used telephone or questionnaire follow-up, either as standard or to obtain information from non-attenders at

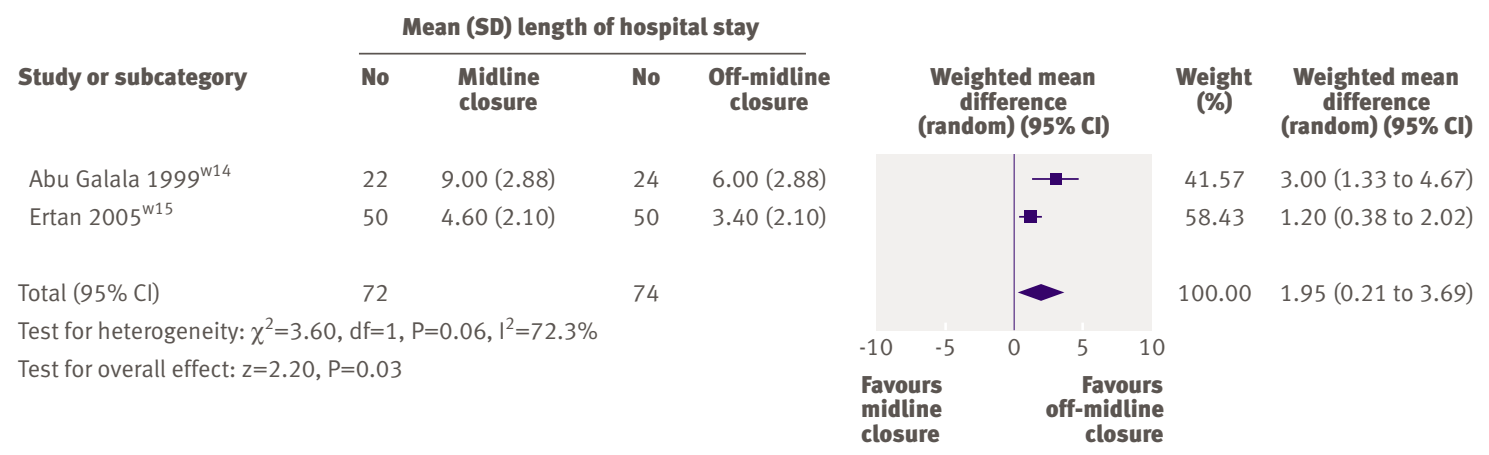




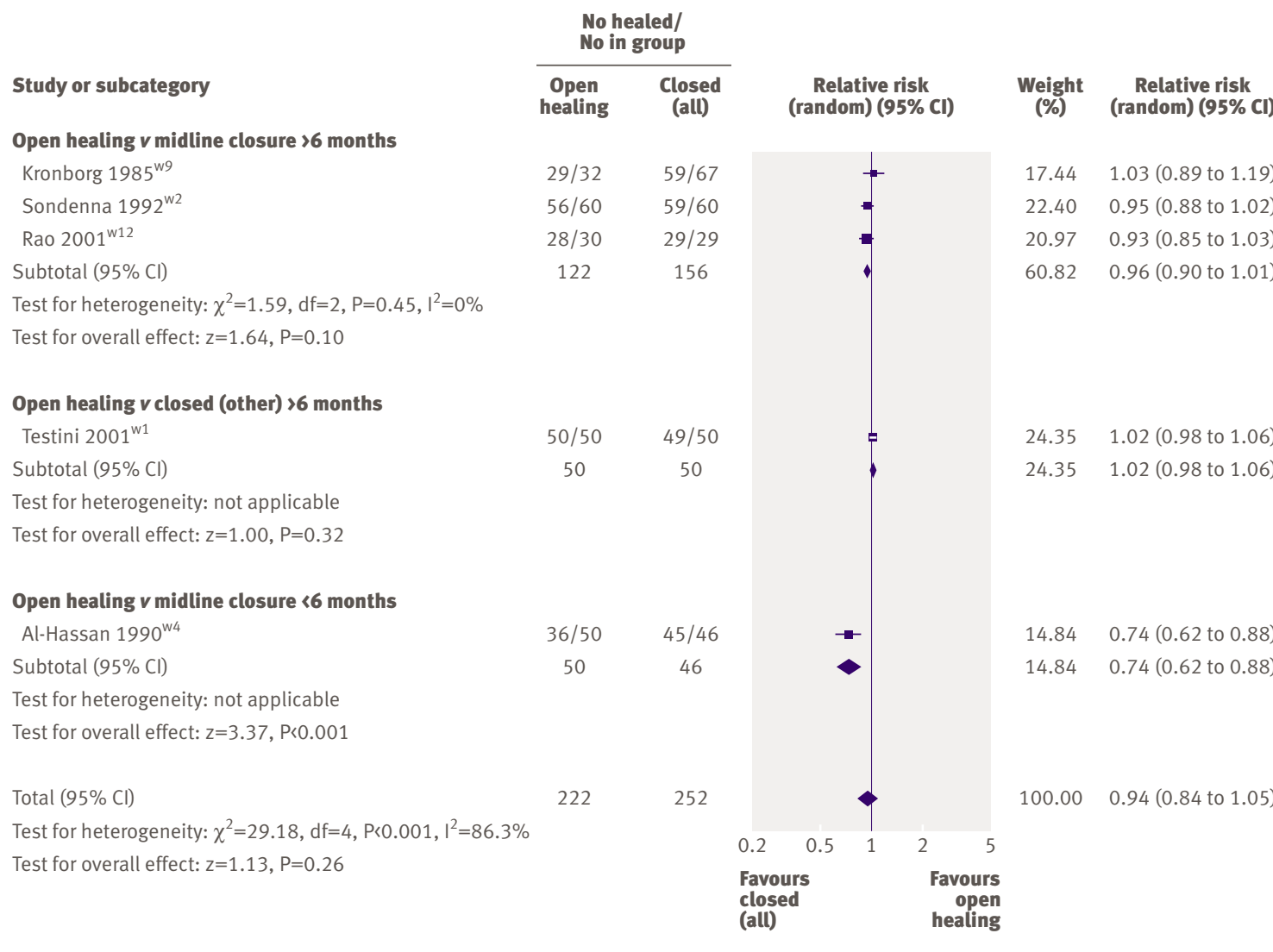

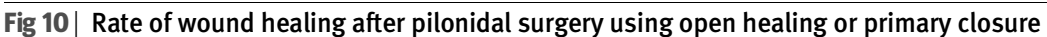

clinic review. Therefore it is possible that a small number of patients with macroscopic but asymptomatic recurrence would not be detected using such assessments.

\section{Implications for practice}

Wounds heal more quickly after primary closure and return to work is sooner than with open healing but at the expense of an increased risk of sinus recurrence. It would seem that treatment type should still be based on surgeon and patient preference, with consideration of the patients' goals for therapy.

Off-midline closure rather than midline closure showed benefit for most clinical and patient outcomes. These data are limited in some areas, although for outcomes where a reasonable degree of evidence has accrued the lack of heterogeneity is reassuring. Available data suggest that off-midline closure should become standard management for pilonidal sinus when primary closure is the desired surgical option.

\section{Implications for research}

No full economic evaluation has been undertaken of the treatment for pilonidal sinus and further research is needed, particularly given the incidence of disease and economic productivity of the young adult population mostly affected by this condition.

We found few data on pain scores or patient satisfaction by type of surgical procedure. Further investigation into surgical techniques for the treatment and management of pilonidal disease is required. Evidence is still lacking as to whether open healing or primary closure methods should be used. Research should focus on well designed, high quality clinical trials; studies should be adequately powered, possibly by way of multicentre recruitment, and should use standardised protocols for assessment of infection, complications, pain, satisfaction, costs, and recurrence. Primary outcomes are time to wound healing, surgical site infection, and recurrence. Adequate follow-up with clinical assessment would be desirable to accurately detect true recurrence. Finally, studies should

\section{WHAT IS ALREADY KNOWN ON THIS TOPIC}

Pilonidal sinus is common and associated with considerable morbidity in young adults

Surgical management offers the best chance of cure, but surgical techniques have limitations and optimal treatment is unclear

\section{WHAT THIS STUDY ADDS}

After pilonidal surgery wounds heal quicker with primary closure than with open healing but risk of recurrence is increased

The suture line should lie off the midline to ensure trouble free healing and minimal chance of recurrence 
incorporate health economic techniques to estimate associated costs and benefits of different treatment options.

We thank the Cochrane Wounds Group for their support.

Contributors: All authors were responsible for the study design and revising the manuscript. IJDMCC and JB were responsible for data extraction and analysis and writing the manuscript. PMK provided clinical expertise. IJDMCC is guarantor for the paper.

Funding: JB is funded by Medical Research Council special training fellowship in health services and health of the public research. Competing interests: None declared.

Ethical approval: Not required.

Provenance and peer review: Not commissioned; externally peer reviewed.
1 Sondenaa K, Andersen E, Nesvik I, Soreide JA. Patient characteristics and symptoms in chronic pilonidal sinus disease. Int J Colorectal Dis 1995;10:39-42.

2 McCallum I, King PM, Bruce J. Healing by primary versus secondary intention after surgical treatment for pilonidal sinus. Cochrane Database Syst Rev

2007;(4):CD006213.Doi:10.1002/14651858.CDoo6213.pub 2.

3 Higgins JPT, Green S, eds. Cochrane Handbookfor Systematic Reviews of Interventions Version 4.2.5 [updated May 2005]. The Cochrane Collaboration, 2005. www.cochrane-handbook.org.

4 Petersen S, Koch R, Stelzner S, Wendlandt T, Ludwig K. Primary closure techniques in chronic pilonidal sinus: a survey of results of different surgical approaches. Dis Colon Rectum 2002;45:1458-67.

Accepted: 25 February 2008 